\title{
Intravascular tissue reactions induced by various types of bioabsorbable polymeric materials: correlation between the degradation profiles and corresponding tissue reactions
}

\author{
Ichiro Yuki • Naoyuki Uchiyama • Yuichi Murayama • Yih-Lin Nien • Daniel Lee • \\ Masaki Ebara • Akira Ishii • Alexander Chiang • Harry V. Vinters • \\ Ichiro Nishimura $\cdot$ Benjamin M. Wu $\cdot$ Fernando Vinuela
}

Received: 24 October 2009 / Accepted: 4 January 2010/Published online: 10 February 2010

(C) The Author(s) 2010. This article is published with open access at Springerlink.com

\begin{abstract}
Introduction Several different bioabsorbable polymeric coil materials are currently used with the goal of improving treatment outcomes of endovascular embolization of intra-
\end{abstract}

I. Yuki $\cdot$ N. Uchiyama $\cdot$ Y. Murayama $\cdot$ Y.-L. Nien $\cdot$ D. Lee $\cdot$

A. Ishii $\cdot$ A. Chiang $\cdot$ F. Vinuela

Division of Interventional Neuroradiology,

Department of Radiological Sciences, David Geffen

School of Medicine, University of California Los Angeles,

Los Angeles, CA, USA

Y. Murayama $\cdot$ M. Ebara

Department of Neurosurgery, The Jikei University of Medicine,

Tokyo, Japan

H. V. Vinters

Department of Pathology and Laboratory Medicine, David Geffen School of Medicine, University of California Los Angeles,

Los Angeles, CA, USA

I. Nishimura $\cdot$ B. M. Wu

School of Dentistry, Weintraub Center for Reconstructive

Biotechnology, University of California Los Angeles,

Los Angeles, CA, USA

B. M. Wu

Department of Bioengineering,

University of California Los Angeles,

Los Angeles, CA, USA

I. Yuki $(\bowtie)$

Division of Interventional Neuroradiology, UCLA Medical Center and David Geffen School of Medicine at UCLA,

10833 Le Conte Avenue,

Los Angeles, CA 90095-1721, USA

e-mail: iyuki@mednet.ucla.edu

H. V. Vinters

Department of Neurology, David Geffen School of Medicine, University of California Los Angeles,

Los Angeles, CA, USA cranial aneurysms. However, little is known about the correlation between polymer degradation profiles and concomitant tissue responses in a blood vessel. The authors describe in vitro degradation characteristics of nine different polymeric materials and their corresponding tissue responses induced in rabbit carotid arteries.

Methods Mass loss and molecular weight loss of nine commercially available bioabsorbable sutures were evaluated in vitro up to16 weeks. The same nine materials, as well as platinum coils, were implanted into blind-end carotid arteries $(n=44)$ in rabbits, and their tissue reactions were evaluated histologically 14 days after the implantation.

Results Five of the nine polymers elicited moderate to strong tissue reactions relative to the remaining materials. While polymer mass loss did not correlate with their histologic findings, polymers that showed a faster rate of molecular weight loss had a tendency to present more active tissue reactions such as strong fibrocellular response around the implanted material with a moderate inflammatory cell infiltration. Maxon exhibited the fastest rate of molecular weight loss and poly-l-lactic acid the slowest.

Conclusions The rate of molecular weight loss may be an important factor that is associated with the degree of bioactivity when bioabsorbable polymers are implanted into blood vessels. For further quantitative analysis, additional experiments utilizing established aneurysm models need to be conducted.

Keywords Aneurysm - Endovascular treatment . Bioabsorbable polymeric materials $\cdot$ Degradation properties

\section{Purpose}

Bioabsorbable polymeric material coil was originally developed based on the concept that polymer-induced mild 
inflammatory responses elicit better thrombus organization in treated aneurysms [1]. Currently used polymer-coated platinum coils are made of either polyglycolides (PGA) or copolymer of polylactides/polyglycolides (PLGA) [2-4]. PGA/PLGA are among the most commonly used copolymers for bioabsorbable sutures, with well-documented usage in other biomedical applications [5-11]. Although there are several reports describing their in vivo degradation property in subcutaneous tissue in different animals [1214], very few studies have been conducted to evaluate tissue responses observed in blood vessels. In particular, the relationship between polymer degradation property and its corresponding tissue reactions in intraluminal thrombus has not been fully described. Indeed, the knowledge is crucial to control the biological effect induced by the polymeric materials in a treated aneurysm, and consequently, it may contribute to further improvement of the current coil devices for aneurysm treatment.

This study was aimed at elucidating the relationship, if any, between degradation profile of nine different commercially available bioabsorbable polymers and their concomitant tissue responses observed in partially thrombosed vessels. As a first step, in vitro analysis was performed using two readily quantifiable parameters, "mass loss" and "molecular weight loss," to characterize polymer degradation. Next, the nine different polymers, as well as platinum coils, were implanted in the blind-end carotid arteries in rabbits, and their intraluminal tissue responses were evaluated. Special attention was given to the factors that may enhance thrombus organization in the partially thrombosed arteries.

\section{Methods}

In vitro analysis and selection of bioabsorbable polymeric coil materials

The nine bioabsorbable polymers or copolymers, most of which are commercially used surgical sutures, were processed into sterilized short segments under the good manufacturing practice conditions. They were (1) Polysorb (US Surgical, CT, USA), $10 \%$ polyglycolic acid $/ 90 \%$ polylactic acid with coating of glycolide and $\varepsilon$-caprolactone (Surgical suture material. It is also the material used in the coating of Matrix ${ }^{\circledR}$ detachable coil.); (2) 25/75 PLGA (A synthesized polymer thread specifically created for the experiment.); (3) BondekPlus (Teleflex Medical Mansfield, MA, USA; 100\% PGA (Surgical suture material. It is also the same polymeric material used in the Cerecyte ${ }^{\circledR}$ coil material.)); (4) Monocryl (Ethicon, Somerville, NJ, USA) 75\% PGA/ $25 \% \varepsilon$-caprolactone (surgical suture material); (5) Biosyn (US Surgical, CT, USA), $60 \% \mathrm{PGA} / 26 \%$ trimethylene carbonate $/ 14 \% p$-dioxanone (surgical suture material); (6) Maxon (US Surgical, CT, USA), 67\% PGA/33\% trimethylene carbonate (surgical suture material); (7) 75/25 PLGA (A synthesized polymer thread specifically created for the experiment); (8) 10/90 PLGA (A synthesized polymer thread specifically created for the experiment); and (9) poly-l-lactic acid (PLLA; A synthesized polymer thread specifically created for the experiment), respectively. Thickness of the coils vary depending on each material $(0.480-0.760 \mathrm{~mm})$.

In vitro evaluation of these nine polymers was conducted as follows. A $20 \mathrm{mg}$ of each coil was immersed in $10 \mathrm{ml}$ of phosphate-buffered saline (PBS) at $37^{\circ} \mathrm{C}(\mathrm{pH}$ 7.4). Samples were collected at $2,4,8,12$, and 16 weeks. In vitro degradation was determined by measuring mass loss on microbalance and molecular weight using gel permeation chromatography (GPC). For GPC, 0.25 wt.\% of each material was dissolved in chloroform or hexafluoroisopropanol, filtered and injected into a Waters Stratagel HT6E column (Mw range 1,000-10,000,000), followed by a Stratagel HT2 column (Mw range 100-10,000), at a flow rate of $0.6 \mathrm{ml} / \mathrm{min}$. Polystyrene standards (Aldrich Co.), with molecular weights of 760 to 2,000,000, were used to obtain GPC calibration curves. Dilute solution viscosity was conducted to confirm GPC findings according to ASTM D2857-95. Poly-(methylmethacrylate) standards were used for calibration. The Mark-Houwink equation was used to estimate molecular weight $(M)$ :

$[\eta]=K M_{w}^{a}$

where $\eta$ represents the intrinsic viscosity (determined experimentally), $K=0.0003$ for PLG, and $a=0.668$ for PLG.

Implantation of polymeric materials into the common carotid artery in rabbit

With the technical assistance of the Boston Scientific Inc., the prepared candidate materials underwent heat treatments, and one of the ends had a rounded edge to prevent the fibers from unraveling after implantations. The samples were sterilized twice by ethylene oxide gas and packed individually.

All animal experiments were conducted in accordance with policies set by the Chancellor's Animal Research Committee and guidelines from the National Institutes of Health. Forty-four carotid arteries in $22 \mathrm{New}$ Zealand white rabbits (body weight, $4-5 \mathrm{~kg}$ ) were used in this study. Each animal was anesthetized with $35 \mathrm{mg} / \mathrm{kg}$ of intramuscularly administered ketamine and $5 \mathrm{mg} / \mathrm{kg}$ xylazine. After the induction of anesthesia, animals were placed on a heating pad to maintain their body temperatures at $37^{\circ} \mathrm{C}$ during the procedure. Every surgical procedure was conducted under sterilized techniques. 
Forty-four surgical implantations of prepared materials were performed in bilateral common carotid arteries (CCAs) of 22 rabbits. The details of the surgical procedure have been reported previously [15]. Briefly, in supine position, a 3-cm midline incision was made in the neck of a rabbit, and bilateral CCAs were exposed. Each CCA was ligated at the distal end of the exposed segment with a 3-0 proline suture, and a 22-G Angiocath ${ }^{\circledR}$ (BD Medical Sandy, UT, USA) was inserted into the CCA in a retrograde fashion. Next, a 2-cm segment of each prepared bioabsorbable polymeric suture or platinum coil was inserted into the artery through the Angiocath. Finally, another ligature with 3-0 proline was placed near the inserting point to fix the implant and to prevent the migration of the implant into the circulation. Figure 1 shows a simplified diagram explaining anatomical relationship after the implantation.

\section{Sample harvest and preparation}

Tissue samples were harvested at postoperative day 14. The harvesting time point was determined based on the previous study [15], in which histologic findings using the same animal model implanted with Polysorb-platinum hybrid coils (PGLA coils) and bare platinum coils were evaluated over four different postoperative time points (days 3, 7, 14, and 30 ), and variable tissue reactions were observed at day 14.

Animals were euthanized with an intracardiac injection of $60 \mathrm{mg} / \mathrm{kg}$ sodium pentobarbital. The previous incisions were reopened, and each arterial segment containing the implanted material was gently removed and fixed in $10 \%$ buffered formalin. All the harvested samples were sent for histologic analysis. The numerical breakdown of the materials implanted in the animals was Maxon $(n=4$

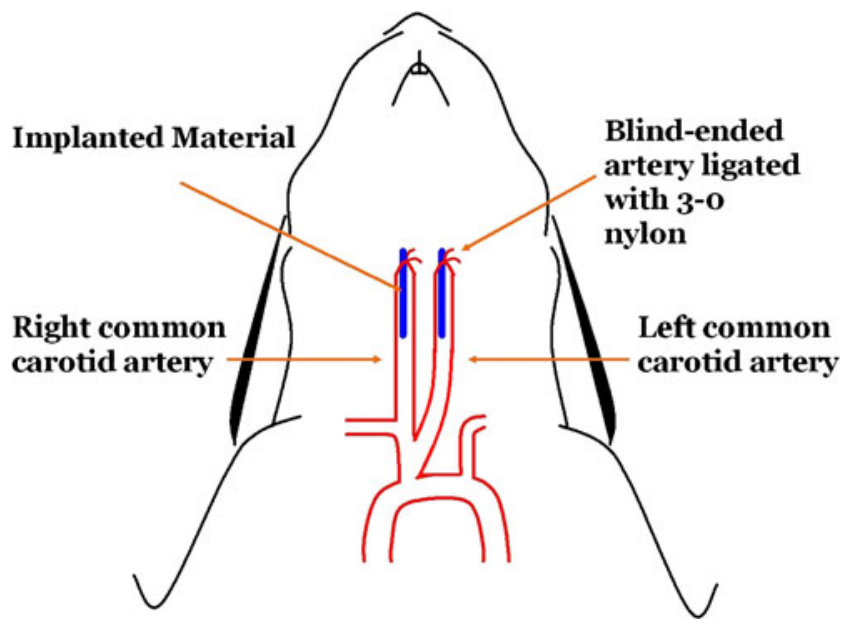

Fig. 1 Animal model used in this experiment: $2 \mathrm{~cm}$ of polymeric suture materials or bare platinum coils was placed in the distal common carotid arteries. Both sides of the distal carotid arteries were ligated with 3-0 proline, and implanted materials were fixed in the blind-ended arterial segments arteries), Polysorb $(n=4)$, Monocryl $(n=4)$, Biosyn $(n=4)$, 25/75 PGLA $(n=4), 75 / 25$ PGLA $(n=4), 90 / 10$ PGLA $(n=$ $4)$, BondekPlus $(n=4)$, PLLA $(n=4)$, and platinum control $(n=4)$. Four sham samples (without implantation) were also evaluated as controls.

Histologic findings

The arterial segments containing platinum were embedded in methylmethacrylate, and the segments containing bioabsorbable polymers were embedded in paraffin. All specimens were sectioned transversely at 2-mm intervals from the distal to the proximal end. The middle third sections were selected for comparing one material to another in order to minimize variability among samples. Sections were stained with hematoxylin and eosin, Masson's trichrome, and Elastic Van Gieson. Special attention was given to the amount, location, and quality of thrombus, inflammatory cells, and fibrous tissues.

Materials used in this study have different braiding patterns and various mechanical properties. Therefore, qualitative histologic analysis, instead of quantitative analysis, was utilized to evaluate the tissue reaction elicited by each material. Every examined sample was categorized into three different groups according to comprehensive histologic findings. "Minimal tissue reaction" was defined as follows. All four specimens showed no remarkable thrombus organization (the thickest part of fibrous tissue formation surrounding the implanted materials was less than $100 \mu \mathrm{m}$ ), and the intraluminal space was predominantly filled with either immature thrombus or fresh red blood cells. "Strong tissue reaction" was defined as follows. All four specimens presented significant fibrous tissue formation surrounding the implanted materials (the thinnest part of organized thrombus around the material is thicker than $100 \mu \mathrm{m}$ ), and the samples also presented remarkable intimal hyperplasia of the vessel wall. "Moderate tissue reaction" is designated for samples that did not completely satisfy either of the former criteria. Three investigators independently reviewed a minimum of five slides from each specimen, and then the interpretations were confirmed by an experienced neuropathologist (H.V.V.).

\section{Results}

Polymer degradation (in vitro analysis)

Figure 2 shows the mass remaining vs. time plots for all nine polymers. Based on the data from mass loss, the degradation behavior of the nine polymer coils can be categorized into four groups: group 1 (Polysorb, 75/25 PGA/PLA, BondekPlus) lost 50\% mass within 1 month; 


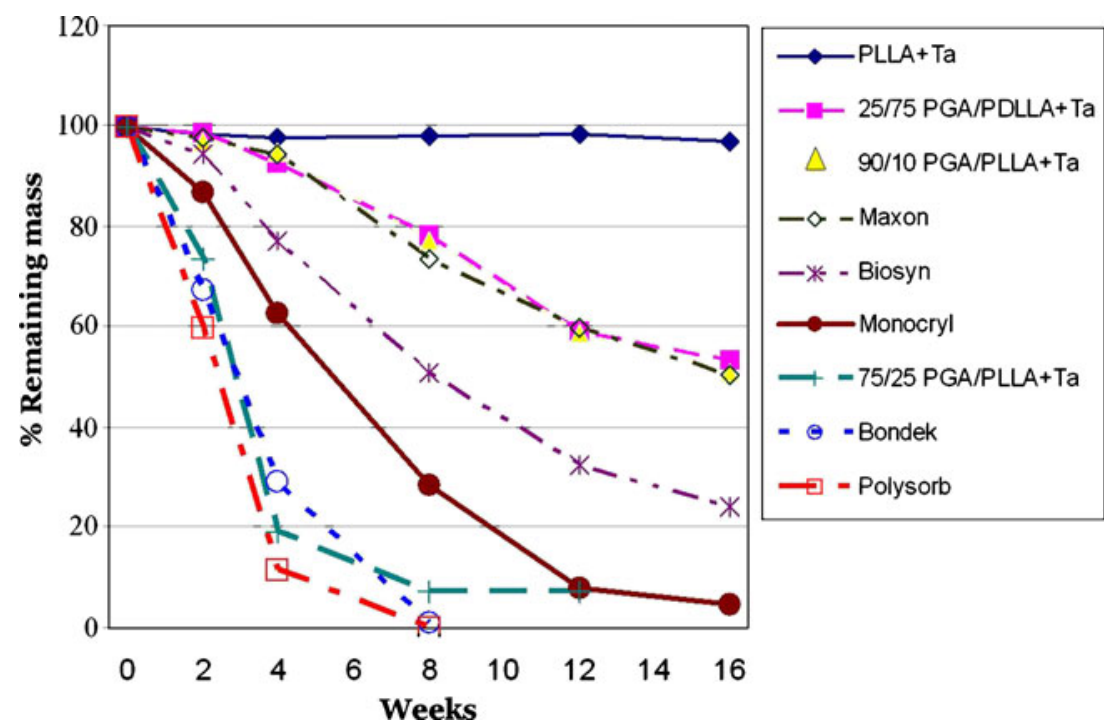

Fig. 2 A graph illustrating the mass loss of each material with time: $20 \mathrm{mg}$ of each coil was immersed in $10 \mathrm{ml}$ of $\mathrm{PBS}$ at $37^{\circ} \mathrm{C}(\mathrm{pH} 7.4)$. Based on the data from mass loss, the degradation behavior of the nine polymer coils can be categorized into four groups: group 1 (Polysorb, 75/25 PGA/PLA, BondekPlus) lost 50\% mass within 1 month; group

group 2 (Monocryl, Biosyn) lost 50\% mass within 2 months; group 3 (25/72 PGA/PLA, Maxon, 90/10 PGA/PLA) lost 50\% mass within 4 months; and group 4 (PLLA) did not lose significant mass during the 4-month period.

Figure 3 shows the molecular weight degradation profile for each polymer. As expected, molecular weight loss precedes mass loss for each coil. In general, there is a general correlation between mass loss and molecular weight loss data in terms of susceptibility to hydrolytic degradation. However, slight differences exist between the relative

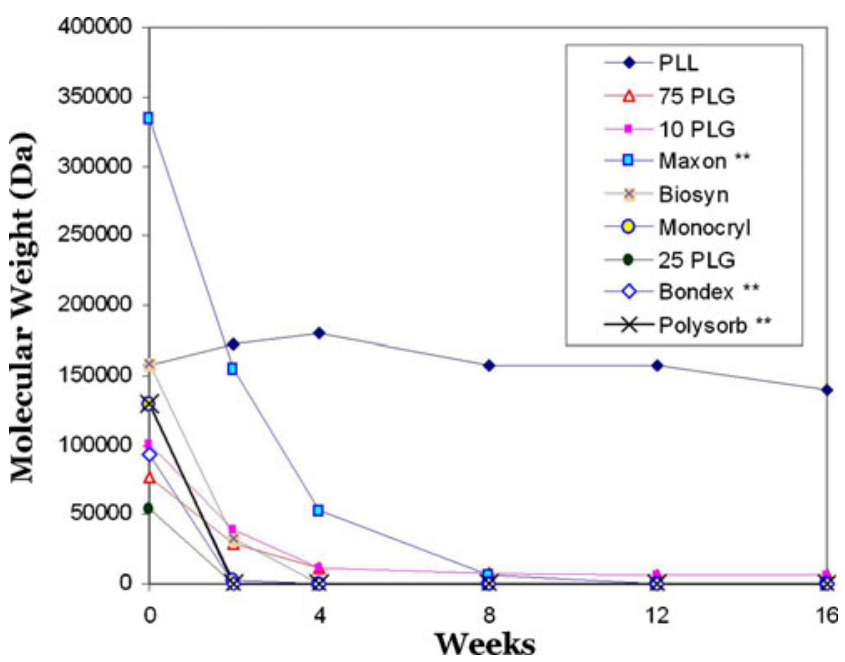

Fig. 3 Molecular weight loss of each material with time: the same procedure for measuring mass loss was used, except that the remaining molecular weight was measured instead of mass. Note that the majority of the materials lost their greatest amount of molecular weight during the initial 2 weeks
2 (Monocryl, Biosyn) lost 50\% mass within 2 months; group 3 (25/72 PGA/PLA, Maxon, 90/10 PGA/PLA) lost 50\% mass within 4 months; and group 4 (PLLA) did not lose significant mass during the 4-month period

order of molecular weight loss and the order of mass loss. Then, we focused on the initial 14 days, where the most prominent rate of molecular weight loss was observed in every material. The degradation behavior of the nine polymer coils was categorized into three groups: fast initial degraders (Maxon, Biosyn, Monocryl, BondekPlus, and Polysorb) lost the greatest amount of molecular weight in the initial 2 weeks; intermediate initial degraders (90:10, 75:25, and 25:75 PGA/PLA) lost less molecular weight in the initial 2 weeks; and slow degraders (PLLA) did not lose significant mass during the initial 2 months.

Fig. 4 Histologic findings of implanted materials in the rabbit carotid arteries (H\&E staining, $\times 20$, except $k)$. $a$ Sham: artery lumen filled with fresh red blood cells. No intimal thickening, inflammatory response, or fibrocellular reaction was observed. $b$ Platinum coil: implanted material did not provoke a substantial fibrous tissue or inflammatory response. $c$ 25/75 PGLA: no significant intimal hyperplasia or cellular response was observed. $d$ PLLA: minimal amount of fibrous tissue covering the material was observed. Neither significant intimal hyperplasia of inner wall nor well-organized thrombus was seen filling the cavity. $e-1$ Monocryl: intimal hyperplasia of the arterial wall and thick fibrocellular tissue around the material were seen. Minimal immature thrombus was observed inside of the material and in the vessel cavity. ( $e-2$ shows higher magnification with $\times 3$ digital zoom). $f-1$ Polysorb: thick fibrocellular tissue surrounding the polymer filament was observed. Luminal cavity was filled with well-developed complex of intimal hyperplasia and organized thrombus surrounding the material. $(f-2$ shows higher magnification with $\times 3$ digital zoom). $g-1$ Maxon: strong fibrocellular response was observed both inside and outside of implanted material and merged into the thickened inner wall. Very little amount of immature thrombus was observed ( $g$-2 shows higher magnification with $\times 3$ digital zoom). $h$ Foreign body giant cell observed in a Polysorb-implanted artery. $(\mathrm{H} \& \mathrm{E}, \times 40)$ : Arrow shows a multinuclear giant cell adjacent to a polymeric material 
Histologic evaluation (in vivo analysis)

The amount of thrombus, inflammatory cells, and fibrous tissue was minimal or absent in control arteries (Fig. 4, a), whereas most of the treated specimens exhibited some degree of thrombus formation and thrombus organization associated with the implanted material. Macrophages and multinuclear giant cells commonly accumulated on the surface of the biomaterials (Fig. 4, h). The absence of a significant neutrophil infiltrate indicated that the leukocyte (mostly monocytic) reaction was considered to be specific to the material and not the result of contaminated (nonsterile) implants. Special attention was given to thrombus organization, luminal spaces, and general inflammatory
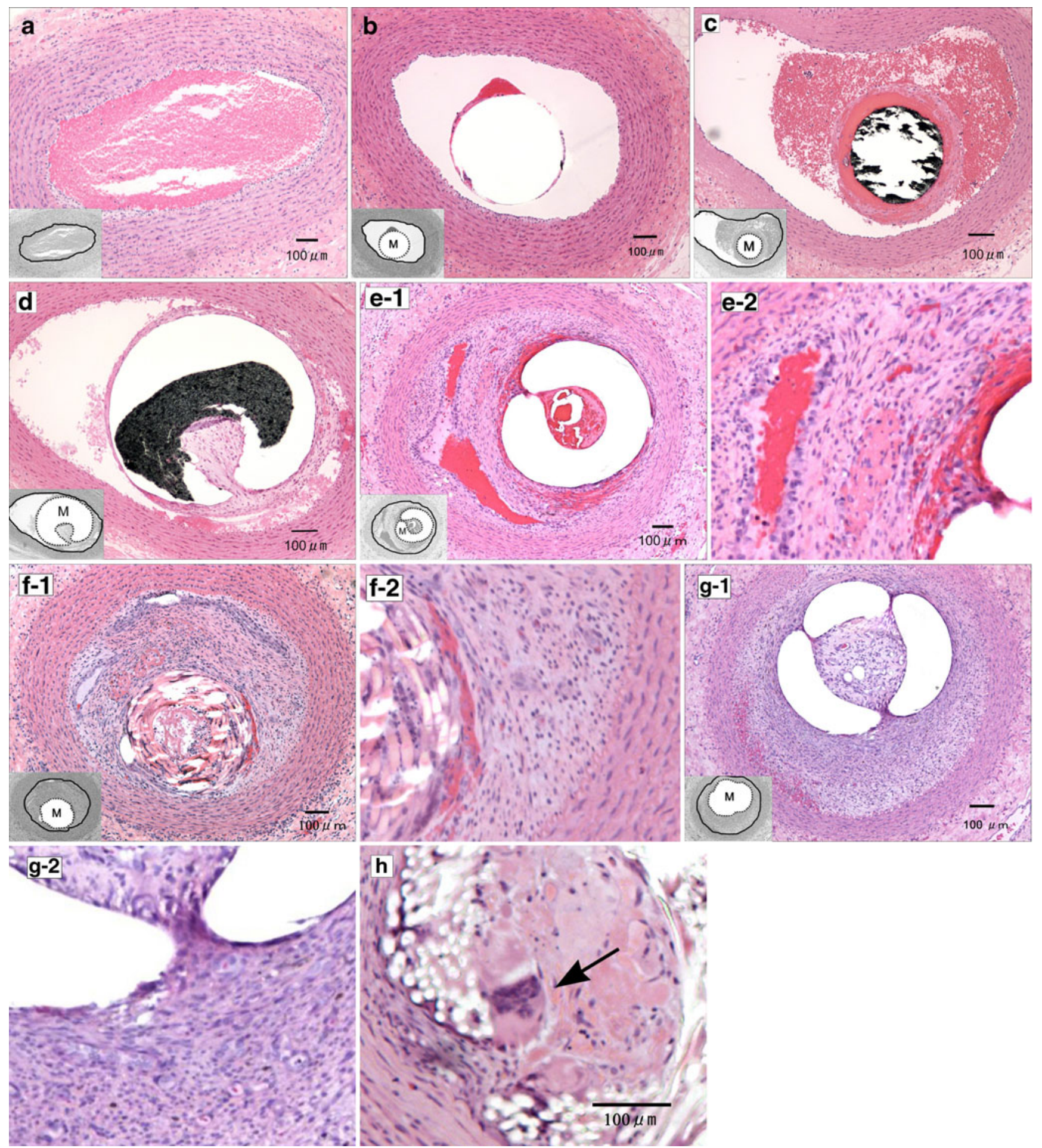
reactions induced by the different biopolymers. (Fig. 4, a-h: figures of histologic findings)

Bare platinum coils (Fig. 4, b), 25/75 PLGA (Fig. 4, c), 75/25 PLGA, and PLLA (Fig. 4, d) elicited very little inflammatory response and fibrocellular transformation. Two out of four specimens treated with Biosyn showed a moderate to strong fibrocellular response around the implanted material with a moderate inflammatory cell infiltration, whereas other two Biosyn-treated specimens did not show such tissue reactions, and minimal tissue reaction remained. Monocryl (Fig. 4, e-1 and e-2) and BondekPlus showed similar histologic findings to Biosyn in producing a moderate fibrocellular response. Polysorb (Fig. 4, f-1 and f-2) demonstrated an intense fibrocellular tissue reaction around the material. Maxon (Fig. 4, f-1 and $\mathrm{f}-2$ ) was also observed to provoke the most intense fibrocellular response of all bioabsorbable polymers/ copolymers examined in this study.

Based on the criteria of tissue reactions described in the "Methods" section, bare platinum, 25/75 PLGA, 75/25 PLGA, and PLLA belonged to the category of "minimal tissue reaction." Biosyn, Monocryl, and BondekPlus were categorized as "moderate tissue reaction" and Polysorb and Maxon as "strong tissue reaction," respectively. These interpretations were confirmed by an experienced neuropathologist (H.V.V.).

Comparison between the results from the material degradation study (in vitro) and histologic evaluations (in vivo)

Although almost no correlation was seen between the "rate of mass loss" and the "tissue reactions," there was a strong correlation between the "rate of molecular weight loss within the first 2 weeks" and the "intensity of tissue reactions elicited by each material." Figure 5 shows the correlation between the implanted materials ( $x$-axis) and their rate of molecular weight loss during first 2 weeks (y-axis). Polymers which exhibited relatively rapid molecular weight loss showed moderate to intense fibrocellular response in the histologic analysis (arrows in the $x$-axis). In contrast, materials with relatively slow rate of molecular weight loss showed minimal tissue reactions in the histologic analysis.

\section{Discussion}

Bioabsorbable polymeric materials and their inducing tissue reaction

Van der Giessen et al. [16] investigated the biocompatibility of five different synthetic polymer stents by placing them in swine coronary artery. These materials had evoked various degrees of inflammatory reaction and fibrocellular proliferation. Chu et al. [12] evaluated the correlation between the composition ratio of PGLA and tissue response. In that study, the 50/50 PGLA copolymer elicited the fastest in vivo degradation and the most intense inflammatory reaction in the subcutaneous tissue of mice.

Although these reports provide a certain degree of information about the tissue reactions induced by bioabsorbable polymeric materials implanted in different animal models, they are not sufficient for investigators to explore new polymer-based coil materials for aneurysm treatment. To further improve the bioactivity of currently used polymeric coils, more detailed information, e.g., factors determining their biological activity, is crucial. Especially, correlation between their degradation properties and the biological reaction induced in the intravascular thrombus has been of considerable interest.

Correlation between "molecular weight loss of each material" and "tissue response"

In general, the degradation mechanism of implanted biomaterials is explained by chemical degradation via hydrolysis or enzyme-catalyzed hydrolysis [11, 17]. The intrusion of water triggers chemical polymer degradation, leading to the creation of oligomers and monomers. During this process, the length of each polymeric chain significantly shortens, and the average molecular weight also decreases. Subsequently, the progressive degradation changes the microstructure of the bulk through the formation of pores, via which oligomers and monomers are released. Afterwards, its physicomechanical property is changed, and mass loss occurs.

Our results showed that molecular weight loss of each material always preceded its mass loss. This finding is consistent with the aforementioned theoretical degradation process. It is also interesting that the fast initial degraders (Maxon, Biosyn, Monocryl, BondekPlus, and Polysorb), which were the materials that showed relatively strong tissue reactions, lost the greatest amount of molecular weight in the initial 2 weeks, while mass loss was hardly exhibited during this time period for the same materials. In fact, the mass loss from these products during the first 2 weeks was negligible when compared to the total mass of the polymer. These facts support the hypothesis that it is the low molecular weight products that are released through the surface of the material itself that actually induce biological responses. There is another fact that strongly supports this hypothesis; it is the prominent correlation between the rate of molecular weight loss of each material and the intensity of tissue reaction observed in the histologic analysis. As shown in Fig. 5, every material presenting moderate to 
Rate of Molecular Weight Loss (First 14 Days) and Histologic Reaction

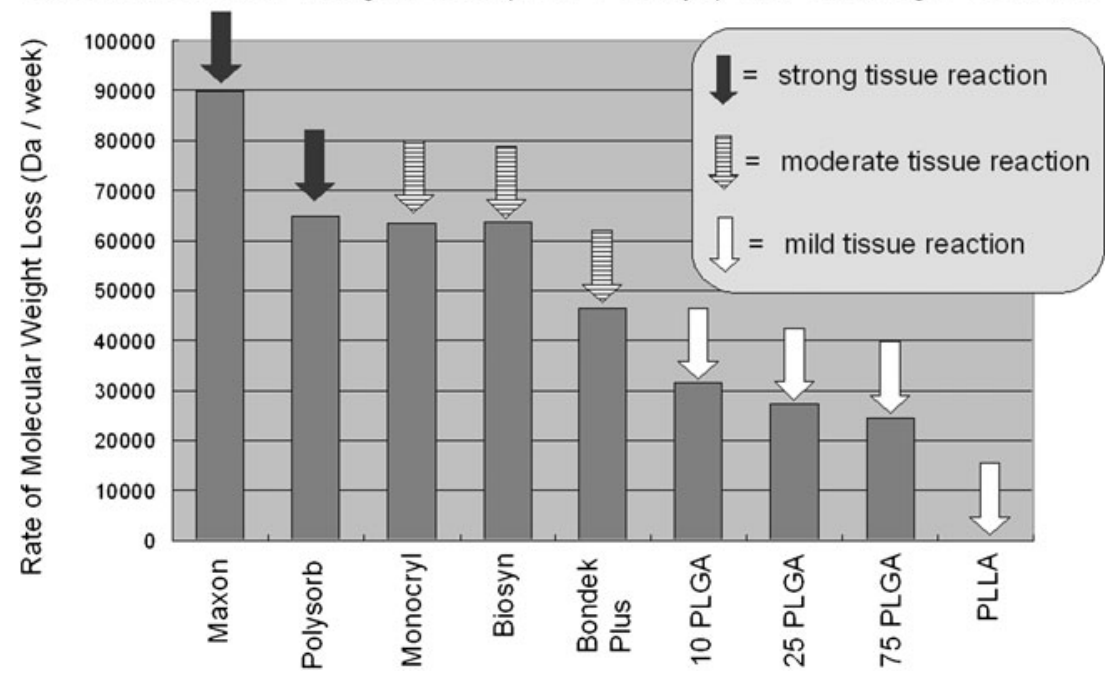

Fig. 5 A bar graph illustrating the correlation between rate of molecular weight loss and histology results: implanted materials $(x-$ axis) and their molecular weight loss in the initial 2 weeks (y-axis) were shown. Arrows indicate tissue reactions induced by each material

intense inflammatory/fibrocellular responses in a blood vessel demonstrated relatively rapid molecular weight loss.

Technical limitations of this study

Although the animal model and the materials used in this study appeared to be very reproducible and consistent, there were several technical limitations in this study. Firstly, although every polymeric material used in this study was composed of PLA, PGA, or their copolymer as a main component, some materials contain other polymeric components (e.g., $\varepsilon$-caprolactone) intermixed with the main component to improve their mechanical properties [18-20]. Since the chemical properties of those additional components could not be analyzed separately, potential influences from those factors should be taken into consideration. Secondly, different braiding pattern of each tested material may have influenced tissue reactions induced by each material. For instance, BondekPlus and Polysorb used in this study were manufactured with braided multifilament whereas the other polymers were made with monofilaments.

Another limitation is related to the animal model used in this study. As described above, each material evaluated in this study was located in the distal portion of a blind-ended arterial segment. Although the sites of implantation showed strong tendency to be thrombosed because of blood flow stagnation, the remaining hemodynamic effect may have influenced the process of thrombus formation/thrombus organization at the proximal end of each material. Therefore, to minimize variability among samples, the middle in the rabbit carotid artery. Note the materials that induced moderate to strong tissue reaction in this study also belonged to the group of the materials that showed relatively rapid molecular weight loss in the initial 2 weeks

third sections were always selected for comparative analysis. We also acknowledge that biological reaction in an open vessel is expected to be different, and the result of this study does not necessarily represent the tissue reaction in such condition.

At last, this study is merely a scientific observation in a simple animal model, and it does not necessarily correlate the treatment outcomes of the patients who were treated with coil materials coated with bioabsorbable polymeric materials. Eventually, for more precise quantitative analysis, additional experiment utilizing other established animal models that have a minimal influence of hemodynamic effect needs to be conducted. In other words, to perform further investigations for preclinical assessment, the selected materials need to be processed into coil products, and they needs to be tested in already-established experimental aneurysm models.

\section{Conclusion}

Mass loss and molecular weight loss of nine commercially available bioabsorbable polymeric sutures were evaluated in vitro up to 16 weeks. The same nine materials, as well as bare platinum coils, were implanted into blind-end carotid arteries in rabbits, and histologic evaluation was performed. Rate of molecular weight loss of each bioabsorbable polymeric material appears to be an important factor that is associated with the intensity of polymer-induced tissue reactions. The animal model used in this study provides us valuable information as initial screening process for new 
bioactive-polymeric materials. However, for further quantitative analysis, additional experiments utilizing other established animal models need to be conducted.

Conflict of interest statement The polymeric materials used in the study were provided by Boston Scientific Inc.

Open Access This article is distributed under the terms of the Creative Commons Attribution Noncommercial License which permits any noncommercial use, distribution, and reproduction in any medium, provided the original author(s) and source are credited.

\section{References}

1. Murayama Y, Vinuela F, Tateshima S, Gonzalez NR, Song JK, Mahdavieh $\mathrm{H}$ et al (2002) Cellular responses of bioabsorbable polymeric material and Guglielmi detachable coil in experimental aneurysms. Stroke 33(4):1120-1128

2. Murayama Y, Tateshima S, Gonzalez NR, Vinuela F (2003) Matrix and bioabsorbable polymeric coils accelerate healing of intracranial aneurysms: long-term experimental study. Stroke 34 (8):2031-2037

3. Pierot L, Flandroy P, Turjman F, Berge J, Vallee JN, Bonafe A et al (2002) Selective endovascular treatment of intracranial aneurysms using Micrus microcoils: preliminary results in a series of 78 patients. J Neuroradiol 29(2):114-121

4. Ishii A, Murayama Y, Nien YL, Yuki I, Adapon PH, Kim R et al (2008) Immediate and midterm outcomes of patients with cerebral aneurysms treated with Matrix1 and Matrix2 coils: a comparative analysis based on a single-center experience in 250 consecutive cases. Neurosurgery 63(6):1071-1077, discussion 1077-1079

5. Athanasiou KA, Agrawal CM, Barber FA, Burkhart SS (1998) Orthopaedic applications for PLA-PGA biodegradable polymers. Arthroscopy 14(7):726-737

6. Cahill DW, Martin GJ Jr, Hajjar MV, Sonstein W, Graham LB, Engelman RW (2003) Suitability of bioresorbable cages for anterior cervical fusion. J Neurosurg 98(2 Suppl):195-201

7. Cohen S, Yoshioka T, Lucarelli M, Hwang LH, Langer R (1991) Controlled delivery systems for proteins based on poly(lactic/ glycolic acid) microspheres. Pharm Res 8(6):713-720
8. Freed LE, Vunjak-Novakovic G, Biron RJ, Eagles DB, Lesnoy DC, Barlow SK et al (1994) Biodegradable polymer scaffolds for tissue engineering. Biotechnology (N Y) 12(7):689-693

9. Tormala P, Pohjonen T, Rokkanen P (1998) Bioabsorbable polymers: materials technology and surgical applications. Proc Inst Mech Eng [H] 212(2):101-111

10. Langer R, Vacanti JP (1993) Tissue engineering. Science 260 (5110):920-926

11. Langer R (1998) Drug delivery and targeting. Nature 392(6679 Suppl):5-10

12. Chu C-C, Von Fraunhofer JA, Greisler HP (1997) Wound closure biomaterials and devices. CRC, Boca Raton

13. Petas A, Karkkainen P, Talja M, Taari K, Laato M, Valimaa T et al (1997) Effects of biodegradable self-reinforced polyglycolic acid, poly-L-lactic acid and stainless-steel spiral stents on uroepithelium after Nd:YAG laser irradiation of the canine prostate. Br J Urol 80 (6):903-907

14. Robert P, Mauduit J, Frank RM, Vert M (1993) Biocompatibility and resorbability of a polylactic acid membrane for periodontal guided tissue regeneration. Biomaterials 14(5):353-358

15. Ebara M, Yuki I, Murayama Y, Saguchi T, Nien Y, Vinters H et al (2009) A rabbit model for efficacy evaluation of endovascular coil materials. Surg Neurol 72:620-627

16. van der Giessen WJ, Lincoff AM, Schwartz RS, van Beusekom HM, Serruys PW, Holmes DR Jr et al (1996) Marked inflammatory sequelae to implantation of biodegradable and nonbiodegradable polymers in porcine coronary arteries. Circulation 94(7): 1690-1697

17. Vert M, Li SM, Garreau H (1994) Attempts to map the structure and degradation characteristics of aliphatic polyesters derived from lactic and glycolic acids. J Biomater Sci Polym Ed 6(7):639649

18. Makela P, Pohjonen T, Tormala P, Waris T, Ashammakhi N (2002) Strength retention properties of self-reinforced poly L-lactide (SRPLLA) sutures compared with polyglyconate (Maxon) and polydioxanone (PDS) sutures. An in vitro study. Biomaterials 23 (12):2587-2592

19. Pitt CG, Jeffcoat AR, Zweidinger RA, Schindler A (1979) Drug delivery systems. I. The permeability of poly(epsilon-caprolactone), poly(DL-lactic acid), and their copolymers. J Biomed Mater Res 13 (3):497-507

20. Pitt CG, Gratzl MM, Jeffcoat AR, Zweidinger R, Schindler A (1979) Sustained drug delivery systems II: Factors affecting release rates from poly(epsilon-caprolactone) and related biodegradable polyesters. J Pharm Sci 68(12):1534-1538 